\title{
MicroRNA-185 regulates chemotherapeutic sensitivity in gastric cancer by targeting apoptosis repressor with caspase recruitment domain
}

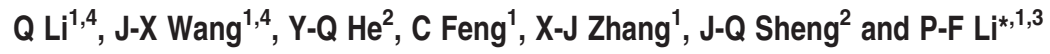

Gastric cancer remains the second leading cause of cancer deaths worldwide. Resistance to chemotherapy is a significant barrier for effective cancer treatment. Here, we identified miR-185 to be a contributor to chemosensitivity in gastric cancer. We observed low levels of miR-185 in gastric cancer cell lines and clinical tissues, compared with gastric epithelium cell line and noncancerous tissues. Furthermore, enforced expression of miR-185 increased the sensitivity of gastric cancer cells to low-dose chemotherapeutic agents, which alone cannot trigger significant apoptosis. Conversely, knockdown of endogenous miR-185 prevented high-dose chemotherapy-induced apoptosis. In elucidating the molecular mechanism by which miR-185 participated in the regulation of chemosensitivity in gastric cancer, we discovered that apoptosis repressor with caspase recruitment domain (ARC) is a direct target of miR-185. The role of miR-185 was confirmed in gastric tumor xenograft model. The growth of established tumors was suppressed by a combination therapy using enforced miR-185 expression and a low dose of anticancer drugs. Finally, we found that RUNX3 (Runt-related transcription factor) was involved in the activation of miR-185 at the transcriptional level. Taken together, our results reveal that RUNX3, miR-185 and ARC regulate the sensitivity of gastric cancer cells to chemotherapy.

Cell Death and Disease (2014) 5, e1197; doi:10.1038/cddis.2014.148; published online 24 April 2014

Subject Category: Cancer

Gastric cancer remains the second leading cause of cancer deaths worldwide. ${ }^{1}$ Chemotherapeutic agents are commonly used for various types of cancer therapy. However, the cytotoxicity and chemotherapy resistance became major barriers to its clinical application. ${ }^{2,3}$ The underlying mechanisms of chemotherapy resistance in gastric cancer are not fully understood.

MicroRNAs (miRNAs) are a class of small noncoding RNAs with 21-25 nucleotides long and negative regulators of target gene by altering mRNA translation or stability. ${ }^{4}$ MiRNAs functionally participated in a wide variety of physiological or pathological processes, including tumor. ${ }^{5}$ Mounting evidence showed aberrant miRNA signatures in human cancers, ${ }^{5}$ implying important roles of miRNAs during tumor progression. A great deal of miRNAs were identified as tumor suppressors; ${ }^{6,7}$ some of these microRNAs have been proven to regulate chemosensitivity. 8,9 The involvement of miRNAs in chemoresistance need to be further investigated.

Apoptosis repressor with caspase recruitment domain (ARC) was initially discovered as an endogenous apoptosis inhibitor in the skeletal muscle and the heart. ${ }^{10}$ It can antagonize both intrinsic and extrinsic apoptosis signaling pathway. ${ }^{11}$ Recent studies showed ARC high expression ${ }^{12,13}$ in many malignant tumors. Our previous work has proved that highly expressed ARC contributed to chemotherapy resistance in cancer cells by targeting the mitochondrial fission machinery. It was also demonstrated that ARC expression was downregulated in cancer cells following doxorubicin treatment, ${ }^{14}$ but the mechanism is largely unknown.

It was reported that phosphorylation ${ }^{15}$ and dephosphorylation $^{16}$ controlled ARC activity. Further studies showed that ARC could be modulated transcriptionally by p53 or Foxo3a. ${ }^{17,18}$ In addition, ARC was downregulated through MDM2-dependent ubiquitination and degradation. ${ }^{19}$ These investigations were mainly performed in cardiomyocytes. Preliminary studies in tumor cells demonstrated that phosphorylation of $A R C^{20}$ was required for its function. However, whether miRNA can regulate ARC in cancer cells is unknown.

The RUNX (Runt-related transcription factor) family consists of three members, RUNX1, RUNX2 and RUNX3. RUNX1 and RUNX2 were essential for hematopoiesis and osteogenesis, respectively. ${ }^{21}$ RUNX3 was identified as a candidate tumor suppressor involved in gastric cancer. ${ }^{22}$ In about $40-60 \%$ of human gastric cancers, loss of RUNX3 expression was observed. ${ }^{22}$ However, the molecular mechanism by which RUNX3 suppressed the growth of cancer cells remains unclear.

\footnotetext{
${ }^{1}$ National Key Laboratory of Biomembrane and Membrane Biotechnology, Institute of Zoology, Chinese Academy of Sciences, Beijing 100101, China; ${ }^{2}$ Department of Gastroenterology, Beijing Military General Hospital, Beijing 100700, China and ${ }^{3}$ College of Medicine, University of Illinois at Chicago, Chicago, IL, USA

${ }^{*}$ Corresponding author: P-F Li, National Key Laboratory of Biomembrane and Membrane Biotechnology, Institute of Zoology, Chinese Academy of Sciences, Beijing 100101, China. Tel: +86 106480 7176; Fax: +86 10 64807176; E-mail: peifli@ioz.ac.cn

${ }^{4}$ These authors contributed equally to this work.

Keywords: miR-185; chemosensitivity; gastric cancer; ARC; RUNX3

Abbreviations: ARC, apoptosis repressor with caspase recruitment domain; RUNX, Runt-related transcription factor; qRT-PCR, quantitative reverse transcription PCR; UTR, untranslated region; ChIP, chromatin immunoprecipitation

Received 19.11.13; revised 30.1.14; accepted 19.2.14; Edited by E Candi
} 
Aberrant miR-185 expression was found in several types of cancers. ${ }^{23}$ In this study, we focused on the function of miR-185 in gastric cancer. We found that miR-185 could sensitize gastric cancer cells to chemotherapy through negatively regulating ARC expression. Besides, miR-185 was upregulated by RUNX3 at the transcriptional level. These results were also confirmed in human gastric cancer samples and xenograft tumor models. Our results identify a novel regulatory pathway for apoptosis involving miR-185 and possibly provide a valuable insight into cancer therapy.

\section{Results}

MiR-185 enhances the sensitivity of gastric cancer cells to chemotherapy. Aberrant expression of miR-185 has been reported in several types of cancers. ${ }^{23}$ To explore the potential role of miR-185 in human gastric cancer, we analyzed 25 pairs of human gastric cancer tissues and matched adjacent noncancerous tissues (Supplementary Table 1). Quantitive analysis revealed that miR-185 levels were significantly reduced in gastric cancer samples, compared with normal gastric tissues (Figure 1a). However, obvious decrease in miR-185 expression in cancer tissues was observed in 20 of 25 patients (Supplementary Figure 1a). In parallel, miR-185 expression was significantly reduced in all five collected gastric cancer cell lines, compared with that in human gastric epithelium cell line GES-1. The endogenous levels of miR-185 were relatively lower in SGC-7901, BGC-823 and MGC-803 (Figure 1b). When exposed to cisplatin $(30 \mu \mathrm{M})$ or doxorubicin $(2 \mu \mathrm{M})$, markedly elevated miR-185 levels were determined in SGC7901 (Figure 1c) and MGC-803 cells (Figure 1d).

The elevation of miR-185 expression induced by cisplatin or doxorubicin treatment led us to consider whether miR-185 was involved in the regulation of anticancer drug-induced apoptosis. To this end, we produced a construct encoding miR-185. Enforced expression of miR-185 was confirmed by quantitative RT-PCR (qRT-PCR) (Supplementary Figure 1b). Overexpression of miR-185 alone had no significant effect on apoptosis (Figures 1e and f), which was consistent with the results of previous study. ${ }^{24}$ To characterize the function of endogenous miR-185 in the apoptotic signaling pathway, which mediated chemotherapy, miR-185 antagomir was transfected into SGC7901. MiR-185 levels were reduced by its specific antagomir. Cisplatin- or doxorubicin-induced apoptosis was attenuated by miR-185 antagomir (Figures 1g-i and Supplementary Figures $1 \mathrm{c}$ and d). A similar result was obtained in MGC-803 cells (Supplementary Figure 1e). Then, we attempted to investigate the influence of miR-185 on cell susceptibility to chemotherapy. Following low-dose cisplatin $(3 \mu \mathrm{M})$ or doxorubicin $(0.2 \mu \mathrm{M})$ treatment in gastric cancer cells, a limited amount of cells undergoing apoptosis was observed. Whereas when we enforced expression of miR-185, the apoptotic cells were significantly increased in response to the same dose of cisplatin or doxorubicin (Figures $1 \mathrm{j}$ and $\mathrm{k}$ and Supplementary Figure 1f). Taken together, these results suggest that miR-185 reduces chemotherapy resistance in gastric cancer.

ARC is a target of miR-185. To elucidate the potential mechanisms by which miR-185 regulates apoptosis, we performed a bioinformatic analysis by TargetScan and found that human ARC mRNA 3'-untranslated regions (UTRs) contained two binding sites for miR-185 (Figure 2a). We measured ARC protein levels in GES-1 and gastric cancer cells. Western blot results indicated that ARC was highly expressed in gastric cancer cells, whereas it was not detectable in GES-1. Endogenous levels of ARC were inversely associated with that of miR-185 in these cell lines (Figure 2b). Then, we attempted to evaluate if miR-185 modulated ARC expression. Enforced expression of miR-185 resulted in an obvious reduction of $A R C$ protein levels, but not in ARC mRNA levels in SGC-7901 and MGC-803 cells (Figure 2c). In contrast, administration of miR-185 antagomir attenuated the decrease of ARC protein levels upon cisplatin (Figure 2d) or doxorubicin (Figure 2e) treatment. Therefore, it seems that miR-185 modulates ARC expression at the posttranscriptional level. To verify whether miR-185 directly targets ARC, we cloned ARC 3'-UTR containing two miR-185 binding sites downstream of the luciferase reporter gene (ARC $3^{\prime}$-UTR-Wt) to examine luciferase translation driven by the 3'-UTR of ARC. Synthesized miR-185 mimic or miRNA mimic control were transfected into HEK-293 cells to perform the luciferase assays. MiR-185 overexpression induced a decrease in the luciferase activity (Figure 2f). Besides, we generated mutated luciferase constructs ARC $3^{\prime}$-UTR-Mut1 and ARC $3^{\prime}$-UTR-Mut2, and mutations were introduced into the two miR-185 binding sites of ARC $3^{\prime}-U T R$, respectively. We found that both the binding sites were responsible for the role of miR-185 in regulating $A R C$ expression (Figure 2f). Simultaneous mutations of two binding sites could nearly abolish the inhibitory effect of miR-185 on luciferase activity (Figure 2f). We further investigated the effect of miR-185 on ARC expression in gastric cancer cells, as shown by luciferase activity. The loss of luciferase expression driven by ARC $3^{\prime}$-UTR upon cisplatin treatment was attenuated by the sitedirected mutations in ARC $3^{\prime}$-UTR (Figure $2 \mathrm{~g}$ ). This reduction of luciferase activity was also attenuated when transfecting miR-185 antagomir (Supplementary Figure 2). Thus, our data indicate that miR-185 is able to target ARC directly.

MiR-185 regulates chemotherapy resistance through ARC. Abundant studies have reported that ARC was expressed at high levels in cancer cells. ${ }^{20}$ There is evidence implicating that ARC may be involved in carcinogenesis and chemotherapy resistance. ${ }^{25}$ After cisplatin or doxorubicin treatment, we found a strong reduction of ARC protein levels, which is contrary to the alteration of miR-185 levels. However, ARC mRNA levels were not reduced as much as its protein levels (Figures $3 a$ and $b$ ). We next explored the role of ARC in gastric cancer chemoresistance. High-dose cisplatin or doxorubicin triggers significant apoptosis. Enforced ARC expression diminished chemotherapyinduced apoptosis (Figures $3 \mathrm{c}$ and d). Whereas knockdown of endogenous ARC enhanced the sensitivity of gastric cancer cells to low-dose cisplatin (Figure $3 e$ and Supplementary Figure 3a). We wondered whether miR-185 regulated chemotherapy sensitivity in gastric cancer cells by targeting ARC. Overexpression of miR-185 promoted lowdose cisplatin-induced cell death. ARC without $3^{\prime}$-UTR (ARC) showed a stronger inhibitory effect on cell death than ARC 

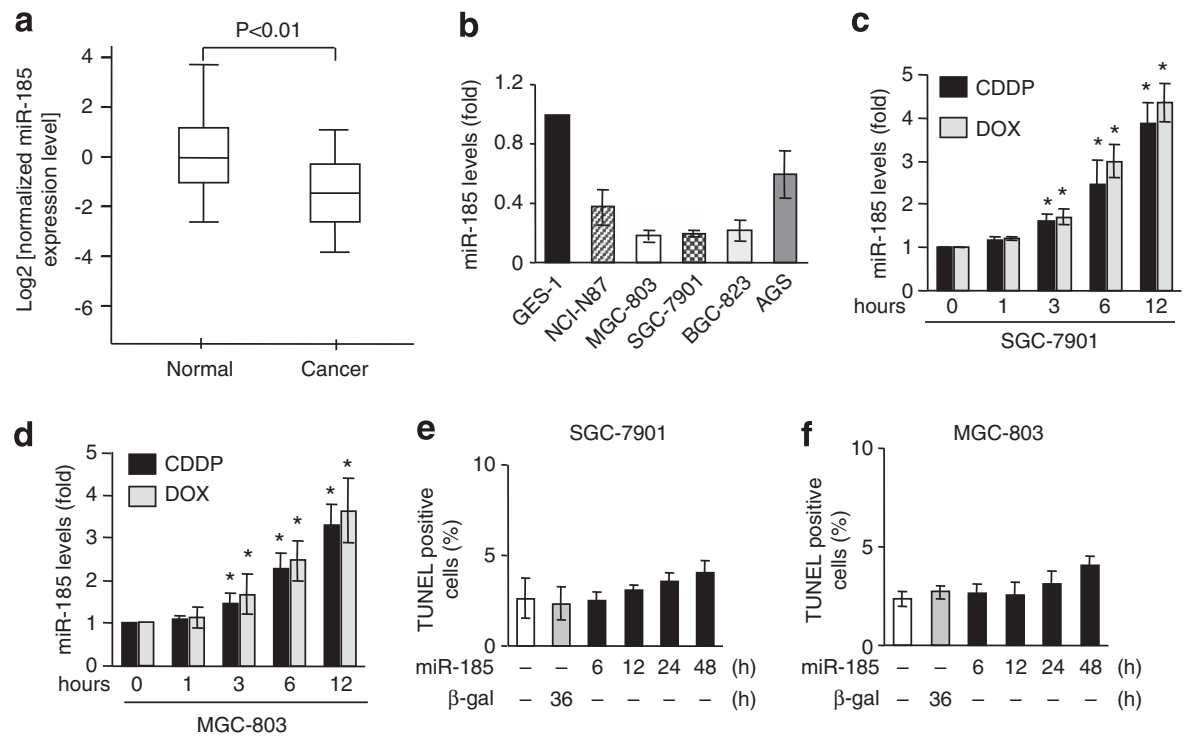

g

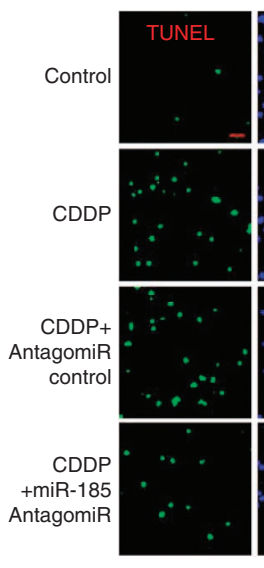

i

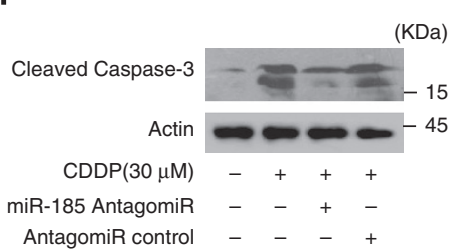

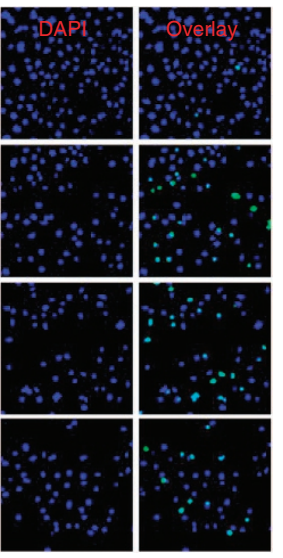

j

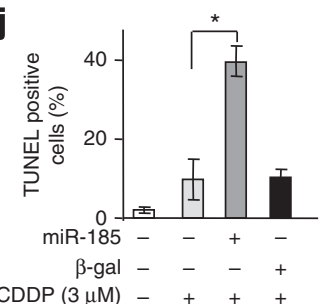

h
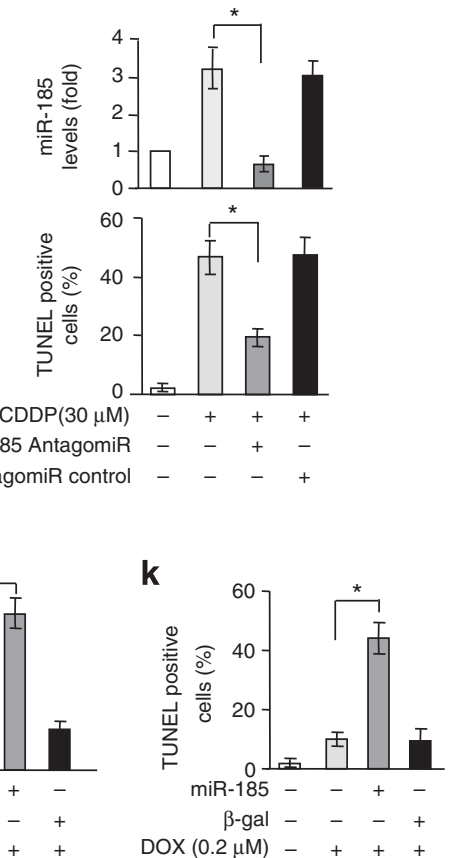

Figure 1 Enforced miR-185 enhances the chemosensitivity of gastric cancer cells. (a) MiR-185 expression levels in 25 pairs of human gastric cancer tissues and matched normal tissues. The boxes represent lower and upper quartiles separated by the median, and the excluding point was marked as a dot. (b) Real-time polymerase chain reaction (PCR) analysis of miR-185 levels in GES-1 and five gastric cancer cell lines. (c and d) MiR-185 levels in SGC-7901 (c) and MGC-803 cells (d) after $30 \mu \mathrm{M}$ cisplatin (CDDP) or $2 \mu \mathrm{M}$ doxorubicin (DOX) treatment as the indicated time. ${ }^{*} P<0.05$ compared with $0 \mathrm{~h}$. (e and $\mathbf{f}$ ) Apoptosis detected by terminal deoxynucleotidyl transferase dUTP nick-end labeling (TUNEL) assay in SGC-7901 (e) and MGC-803 cells (f) overexpressing miR-185. (g-i) Inhibition of endogenous miR-185 using miR-185 AntagomiR (h, top) prevented apoptosis in SGC-7901 treated with $30 \mu \mathrm{M}$ cisplatin for $24 \mathrm{~h}$. Representative images show TUNEL-positive cells (g). Green, TUNEL-positive nuclei; blue, 4',6-diamidino-2-phenylindole (DAPI)-stained nuclei, scale bar $=50 \mu \mathrm{m}$. Expression levels of miR-185 were detected ( $\mathrm{h}$, top). The apoptotic cells were counted ( $\mathrm{h}$, bottom). Cleaved caspase-3 was analyzed by western blot (i). ( $\mathbf{j}$ and $\mathbf{k}$ ) Ectopic expression of miR-185 enhanced apoptosis in SGC-7901 after $3 \mu \mathrm{M}$ cisplatin (j) or $0.2 \mu \mathrm{M}$ doxorubicin (k) treatment for $36 \mathrm{~h}$. Error bars represent S.D. ${ }^{*} P<0.05$

with $3^{\prime}$-UTR (ARC-3'-UTR) in the presence of miR-185 (Figure $3 f$ and Supplementary Figure $3 b$ ). These results reveal that $A R C$ contributes to chemotherapy resistance, which is regulated by miR-185.

MiR-185 is a transcriptional target of RUNX3. The expression of miRNAs can be regulated by transcriptional factors. ${ }^{4}$ To elucidate the mechanism involved in the upregulation of miR-185 upon cisplatin or doxorubicin treatment, we analyzed the promoter sequence of miR-185. Three putative binding sites for RUNX3 were identified (Figure 4a). RUNX3 is a transcriptional factor that has a loss of function in gastric cancer. ${ }^{22}$ Our findings that cisplatin or doxorubicin treatment increased RUNX3 expression 
a

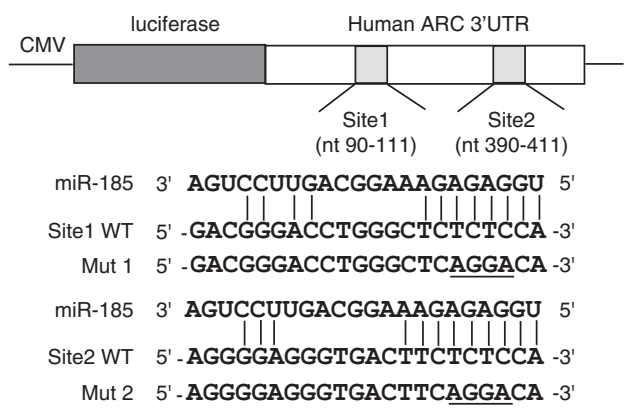

b

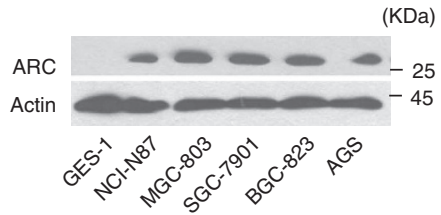

d

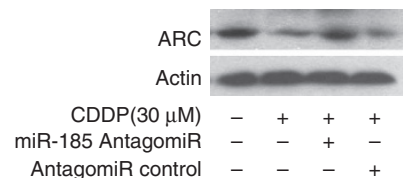

f

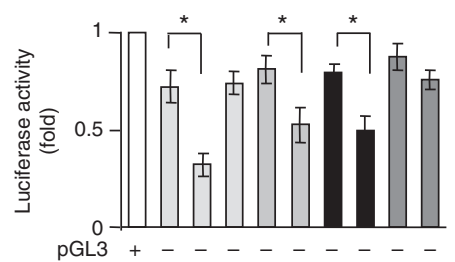

ARC 3'UTR-Wt $\ldots+++\ldots$

ARC 3'UTR-Mut1 - $-\ldots++-\ldots$

ARC 3'UTR-Mut2 - $-{ }_{-}-{ }_{-}+{ }_{-}$

ARC 3'UTR-Mut1+2 $-\ldots+\ldots+-c_{+}+$

miR-185 - $-+\ldots+-+-+$

Mimic control _ $-\ldots+\ldots$ c
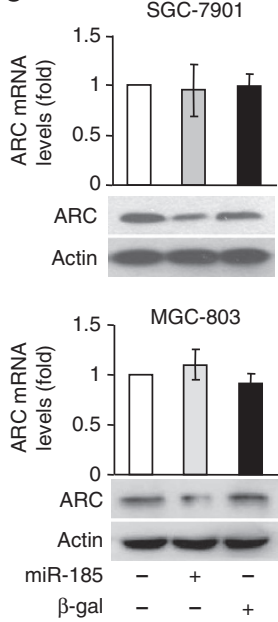

e

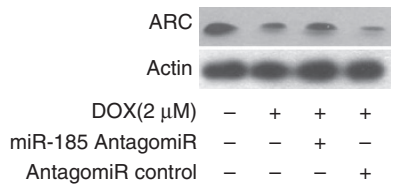

g

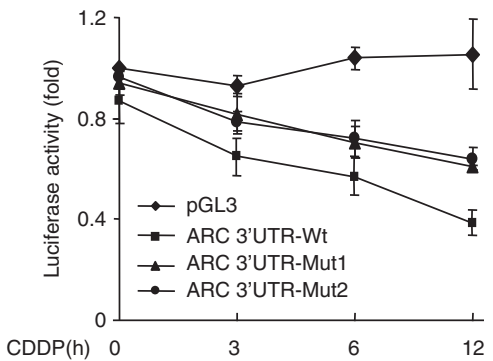

Figure 2 ARC is a direct target of miR-185. (a) Schematic diagram of the reporter constructs containing two putative miR-185 binding sites in human ARC $3^{\prime}-U T R$, mutations introduced into miR-185 binding sites were underlined. (b) Immunoblot of ARC protein in GES-1 and gastric cancer cells. (c) ARC mRNA levels and protein levels in SGC-7901 and MGC-803 cells overexpressing miR-185. (d and e) Knockdown of endogenous miR-185 attenuated decrease of ARC protein levels upon cisplatin (CDDP) (d) or doxorubicin (DOX) (e) treatment for $12 \mathrm{~h}$ in SGC-7901. (f) Luciferase activity detected in HEK-293 transfected with synthesized miR-185 mimic or mimic control, along with luciferase reporter constructs as indicated. (g) Luciferase activity of transfected reporter constructs in $30 \mu \mathrm{M}$ cisplatin-treated SGC-7901. Error bars represent S.D. ${ }^{*} P 0.05$. CMV, cytomegalovirus; $\beta$-gal, $\beta$-galactosidase; Mut, mutated; Wt, wild type

(Figures $4 \mathrm{~b}$ and $\mathrm{c}$ ) led us to consider whether RUNX3 participated in the upregulation of miR-185 expression. To test this hypothesis, the chromatin immunoprecipitation (ChIP) analysis was carried out to test the association of RUNX3 with the promoter of miR-185. It revealed that RUNX3 could directly bind to the binding site 3 (BS3) region but not the binding site 1 (BS1) and 2 (BS2) region in SGC7901 cells treated with cisplatin or doxorubicin (Figure 4d). We next evaluated the transcriptional activity of miR-185 in response to RUNX3. MiR-185 promoter region was cloned upstream of luciferase reporter gene. Overexpression of RUNX3 induced a significant increase of miR-185 promoter activity, and this elevation was reversed by introducing mutations into the BS3 region of miR-185 promoter (Figure $4 \mathrm{e}$ ). We determined that cisplatin or doxorubicin treatment increased miR-185 promoter activity in gastric cancer cells, whereas mutations in BS3 region arrested this elevation (Figure 4f). Finally, we found that enforced expression of RUNX3 led to a remarkable increment of miR-185 expression in SGC-7901 and MGC-803 cells (Figure $4 \mathrm{~g}$ and Supplementary Figure 4). Collectively, our data indicate that miR-185 is directly upregulated by RUNX3 at the transcriptional level.

MiR-185 and ARC are necessary for RUNX3 tumorsuppressing activity. The involvement of miR-185 and $A R C$ in regulating chemotherapy resistance led us to consider whether RUNX3 controls chemosensitivity and apoptosis in gastric cancer through this pathway. Reduced apoptosis of gastric epithelial cells was found in Runx3deficient mice. ${ }^{22}$ Besides, RUNX3 has been shown to 

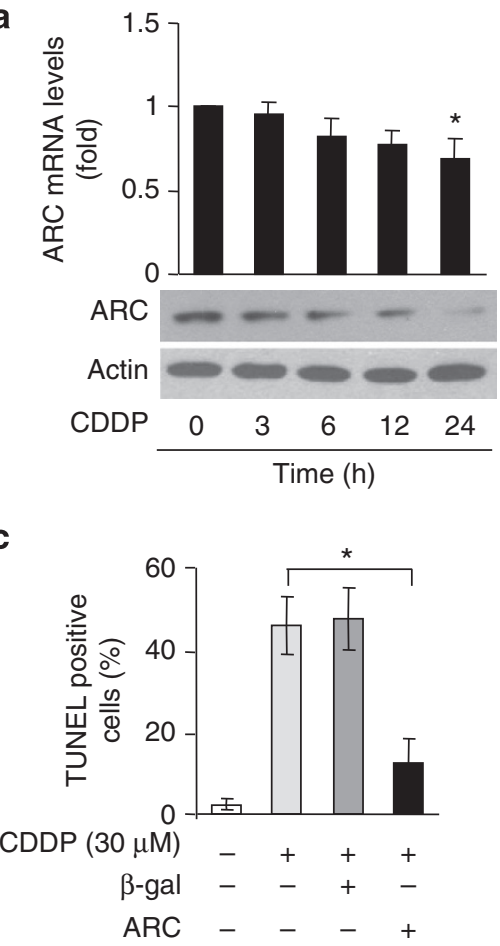

e

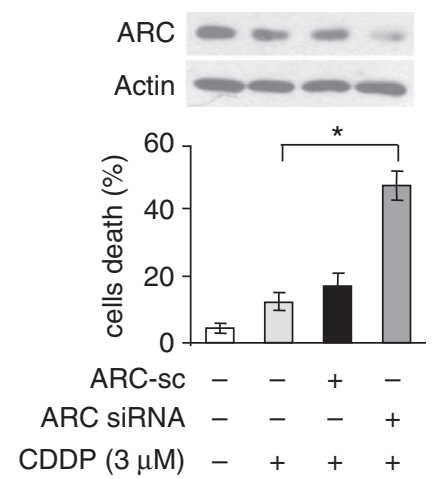

b

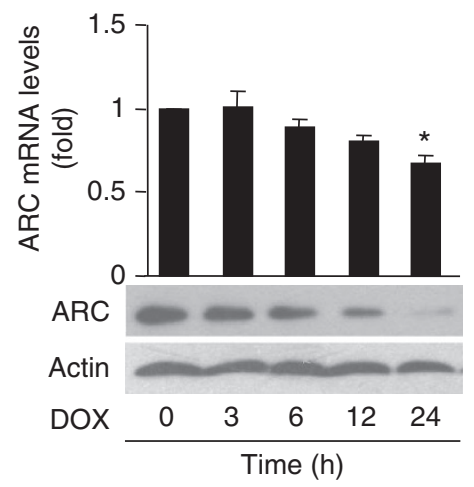

d

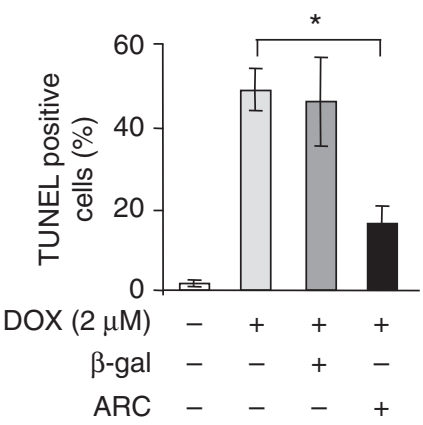

f

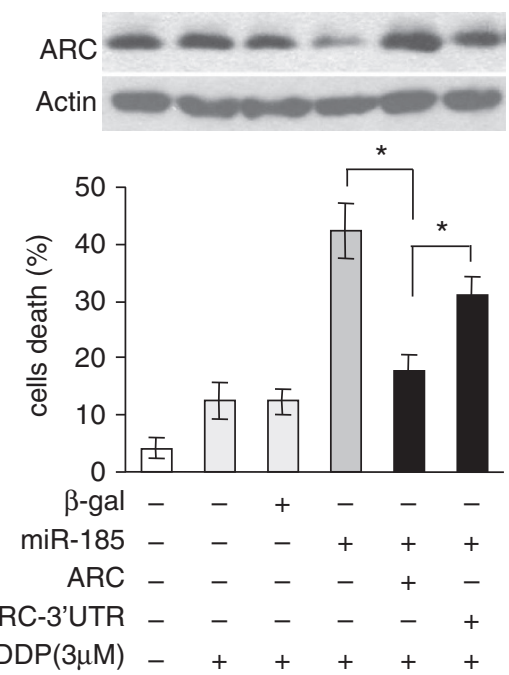

Figure 3 MiR-185 regulates chemotherapy resistance through ARC. (a and b) ARC mRNA levels (top) and protein levels (bottom) in SGC-7901 treated with $30 \mu \mathrm{M}$ cisplatin (CDDP) (a) or $2 \mu \mathrm{M}$ doxorubicin (DOX) (b). ${ }^{*} P<0.05$ compared with $0 \mathrm{~h}$. (c and d) Ectopic expression of ARC prevented apoptosis in SGC-7901 treated with cisplatin (c) or doxorubicin (d) for $24 \mathrm{~h}$. (e) Knockdown of endogenous ARC using its small interfering RNA (siRNA) increased cell death in SGC-7901 treated with cisplatin for $36 \mathrm{~h}$, compared with ARC scrambled form (ARC-sc) group. Cell death was analyzed by trypan blue exclusion. (f) MiR-185 increased cell death in SGC-7901 in response to cisplatin treatment for $36 \mathrm{~h}$, which was prevented by ARC. Error bars represent S.D. ${ }^{*} P<0.05$

mediate TGF- $\beta$-induced apoptosis. ${ }^{26}$ Thus, we first tested whether RUNX3 influenced apoptosis in gastric cancer. An increment of apoptotic cells was observed in SGC-7901 cells overexpressing RUNX3 (Figure 5a). Concomitantly, ARC protein levels, but not its mRNA levels, were strongly reduced (Figure $5 b$ ). Silencing RUNX3 by using its siRNA attenuated the increase of miR-185 levels and reduction of $A R C$ protein levels in response to cisplatin or doxorubicin (Figures $5 \mathrm{c}$ and $\mathrm{d}$ ). Moreover, knockdown of endogenous
RUNX3 diminished chemotherapy-induced cell death in SGC-7901 (Figures 5e and f).

Next, we were interested in determining the effect of RUNX3 on cell susceptibility to anticancer drugs. Strikingly, we found that RUNX3 overexpression led to an elevated amount of cells undergoing cell death, as well as an increase of miR-185 levels in response to low-dose cisplatin (Figure $5 \mathrm{~g}$ ). RUNX3 enhanced cisplatin sensitivity of gastric cancer cells. 


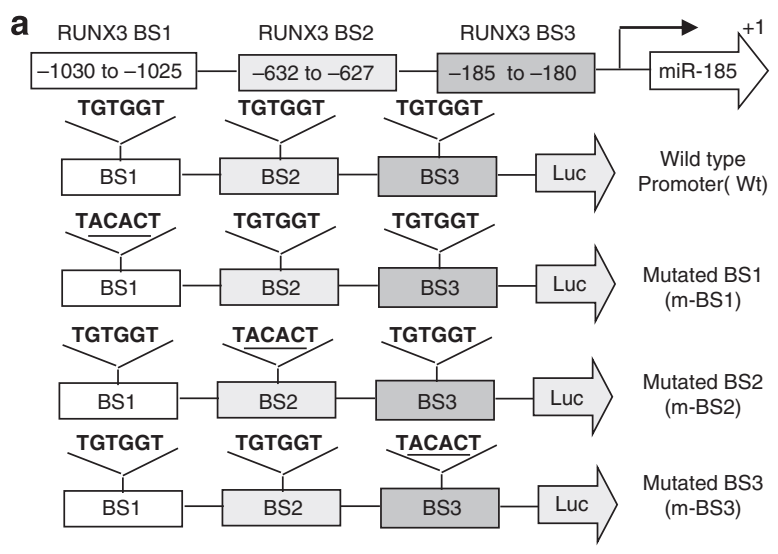

b

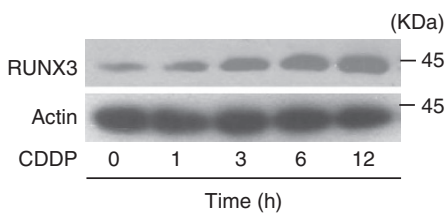

C
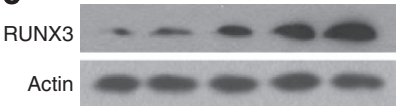

\begin{tabular}{cccccc} 
DOX & 0 & 1 & 3 & 6 & 12 \\
\hline \multicolumn{5}{c}{ Time $(\mathrm{h})$}
\end{tabular}

d

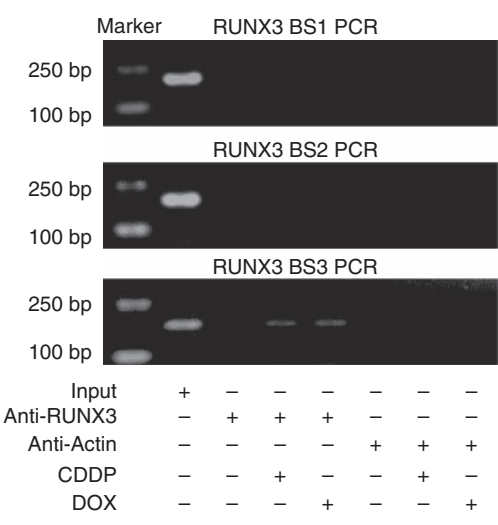

e

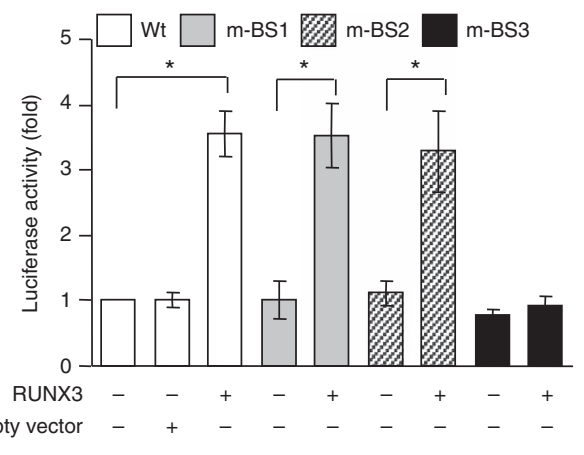

f

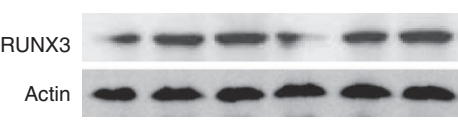

g
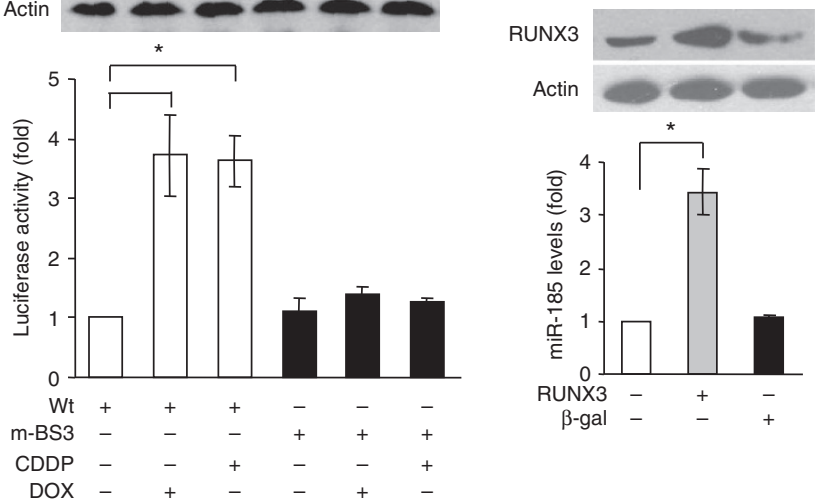

Figure 4 MiR-185 is a transcriptional target of RUNX3. (a) The promoter region of human miR-185 contains three optimal RUNX3 binding sites (BS1, BS2 and BS3). The mutations were introduced into the potential binding sites (m-BS1, m-BS2 and m-BS3). (b and $\mathbf{c}$ ) Immunoblot of RUNX3 protein in SGC-7901 treated with $30 \mu$ M cisplatin (CDDP) (b) or $2 \mu \mathrm{M}$ doxorubicin (DOX) (c). (d) ChIP analysis of RUNX3 binding to miR-185 promoter in SGC-7901 treated with $30 \mu \mathrm{M}$ cisplatin or $2 \mu \mathrm{M}$ doxorubicin for $12 \mathrm{~h}$. (e and f) Luciferase activity of reporter constructs harboring wild-type (Wt) or mutated miR-185 promoter in HEK-293 cells transfected with plasmids encoding RUNX3 (e) or SGC-7901 cells treated with $30 \mu \mathrm{M}$ cisplatin or $2 \mu \mathrm{M}$ doxorubicin for $12 \mathrm{~h}$ (f, bottom). The expression levels of RUNX3 were detected by immunoblot (f, top). (g) Immunoblot of RUNX3 (top) and miR-185 levels (bottom) in SGC-7901 infected with adenoviral RUNX3. Error bars represent S.D. ${ }^{*} P<0.05$

Subsequently, we considered whether miR-185 and ARC contributed to the function of RUNX3 in gastric cancer. Compared with antagomir control, administration of miR-185 antagomir reduced RUNX3-enhanced chemosensitivity (Figure $5 \mathrm{~h}$ and Supplementary Figure $5 \mathrm{a}$ ). This reduction was also observed in the presence of the construct encoding ARC. ARC without $3^{\prime}$-UTR exhibited a stronger inhibitory effect on cell death than ARC with $3^{\prime}$-UTR (ARC-3'-UTR) in the presence of RUNX3 (Figure 5i and Supplementary Figure $5 \mathrm{~b}$ ). These results indicate that RUNX3 mediates chemosensitivity and apoptosis in gastric cancer by modulating miR-185/ARC signaling pathway, although RUNX3 can also target other factors involved in apoptosis. $^{26,27}$ 
a

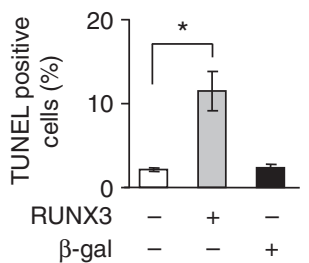

d

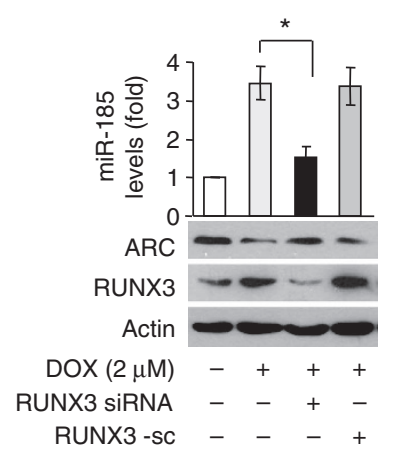

b

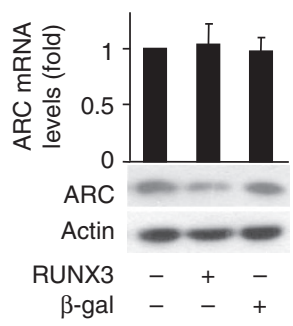

e

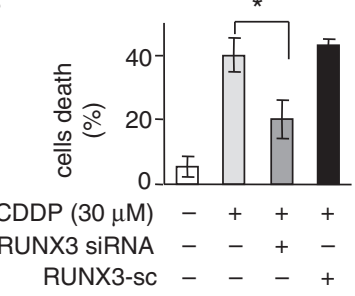

f

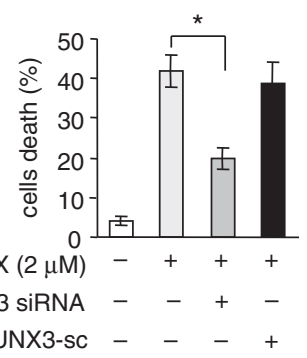

C

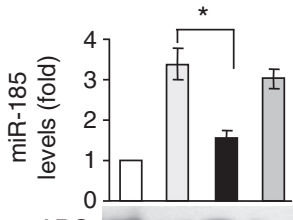

ARC

RUNX3

Actin

$\operatorname{CDDP}(30 \mu \mathrm{M})-+++$

RUNX3 SiRNA $-\quad+-$

RUNX3-sc - $-{ }_{-}+$

g
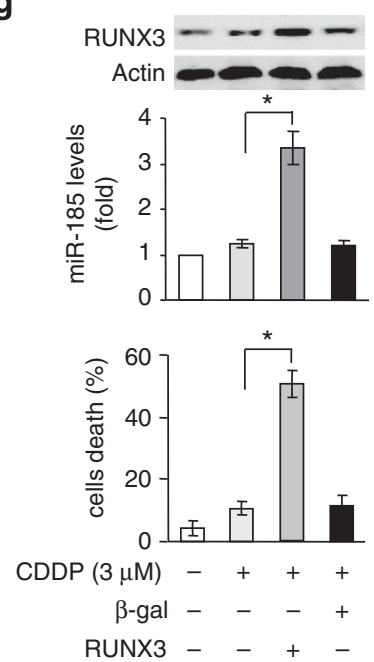

h

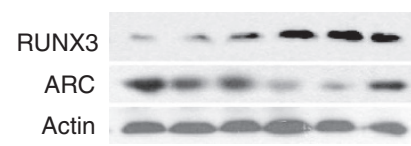

i
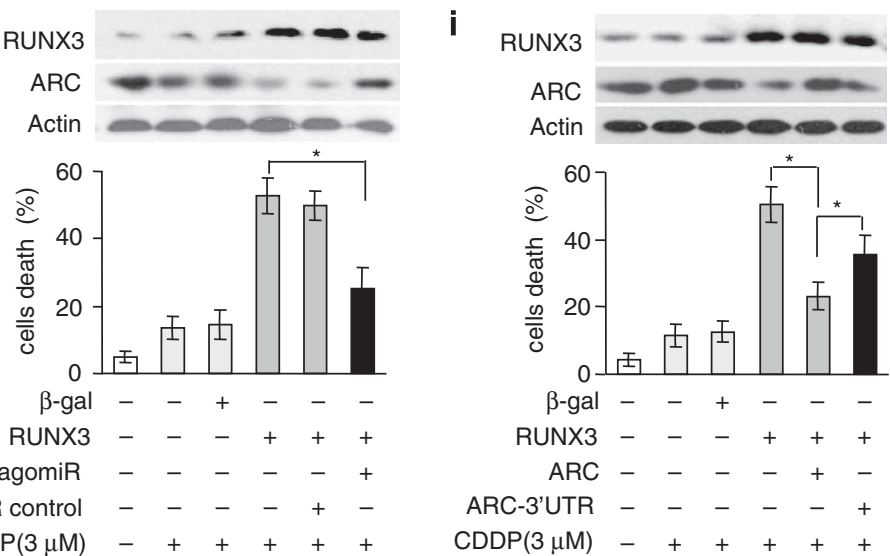

Figure 5 MiR-185 and ARC are necessary for RUNX3 tumor-suppressing activity. (a and $\mathbf{b})$ Determination of apoptosis (a), analysis of ARC mRNA levels (b, top) and protein levels (b, bottom) in SGC-7901-overexpressing RUNX3. (c and $\mathbf{d}$ ) Knockdown of endogenous RUNX3 using its small interfering RNA (siRNA) (bottom) attenuated upregulation of miR-185 expression (top) and downregulation of ARC (bottom) in SGC-7901 following cisplatin (CDDP) (c) or doxorubicin (DOX) (d) treatment for $12 \mathrm{~h}$, compared with corresponding scrambled form (RUNX3-sc) group. (e and f) Knockdown of endogenous RUNX3 attenuated cell death in SGC-7901 treated with cisplatin (e) or doxorubicin (f) for $24 \mathrm{~h}$. (g) Enforced expression of RUNX3 (top) increased miR-185 levels (middle) and cell death (bottom) in SGC-7901 following cisplatin treatment for $36 \mathrm{~h}$. (h and i) RUNX3 increased sensitivity to cisplatin treatment for $36 \mathrm{~h}$ was abolished by miR-185 AntagomiR (h) or ARC (i) in SGC-7901. Error bars represent S.D. ${ }^{*} P<0.05$. $\beta$-gal, $\beta$-galactosidase; TUNEL, terminal deoxynucleotidyl transferase dUTP nick-end labeling

MiR-185 enhances chemotherapeutic effect in vivo. It has been reported that miR-185 suppressed the proliferation of human colorectal cells. ${ }^{28}$ Having demonstrated that miR185 significantly increased the susceptibility of gastric cancer cells to chemotherapy in vitro, we further investigated the role of miR-185 in xenograft models. First, to test whether miR-185 alone could inhibit gastric cancer tumor growth,
SGC-7901 cells stably overexpressing miR-185 or negative control were established. Overexpression of miR-185 was confirmed by qRT-PCR (Supplementary Figure 6a). The stable cells, SGC-7901-miR-185- or SGC-7901-negative control, were injected subcutaneously into nude mice. Xenograft tumor formation was monitored over a 34-day time course. Compared with controls, miR-185 
overexpression resulted in a smaller tumor size (Figure 6a), suggesting that miR-185 suppressed gastric tumor growth in vivo. The mice were killed at day 34. As expected, we found significantly increased miR-185 levels in SGC-7901miR-185-generated tumors, accompanied by the downregulation of ARC (Figure 6b).

Next, we attempted to assess miR-185 therapeutic activity against established tumors. SGC-7901 cells were subcutaneously inoculated into nude mice. When the tumor volumes reached $250-300 \mathrm{~mm}^{3}$, intraperitoneal delivery of doxorubicin and/or intratumoral injection of miR-185 adenovirus were given every other day. Two different doses of doxorubicin, 2 and $4 \mathrm{mg} / \mathrm{kg}$, were used in this study. During 2 weeks of therapy, we followed subcutaneous tumor growth and body weight of the mice. It was found that high-dose chemotherapy appeared as obvious inhibitors of tumor growth, and clear body weight loss indicated the severe toxicity of chemotherapy (Figure 6c and Supplementary Figure 6b). In comparison with high-dose chemotherapy, miR-185 alone or combined with low-dose doxorubicin did not exhibit obvious adverse effect, which was indicated by slight body weight loss (Supplementary Figure 6b). Combination therapy using lowdose doxorubicin and miR-185 successfully restrained the growth of established gastric tumor, as effective as high-dose chemotherapy (Figure 6c). Additionally, quantitative analysis of tumors from combination treatment group showed an elevation of miR-185 levels and remarkable reduction of ARC expression (Figure 6d), which may be contributed to enhanced apoptosis, as assessed by the TUNEL assay (Figure 6e and Supplementary Figure 6c) in xenograft models. These findings were consistent with our obtained results in vitro. To sum up, our data indicate that miR-185 increases gastric cancer chemosensitivity through suppressing $A R C$ expression in vivo.

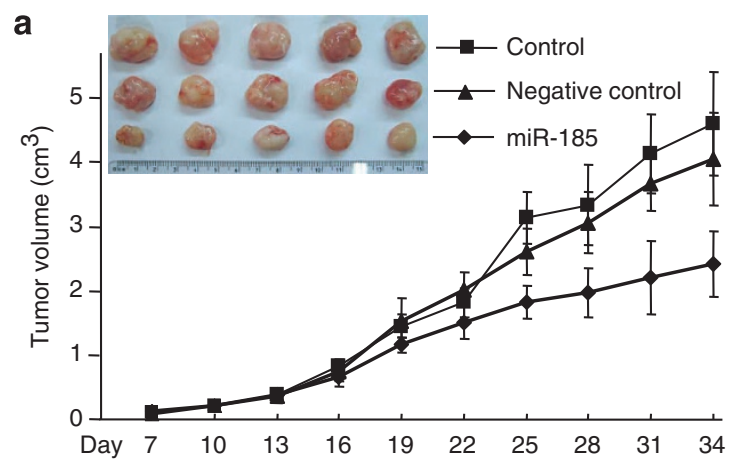

b

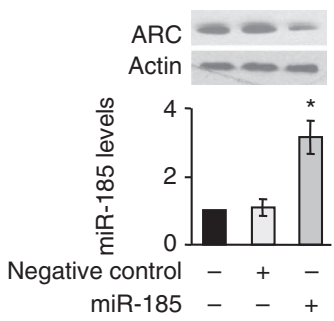

C

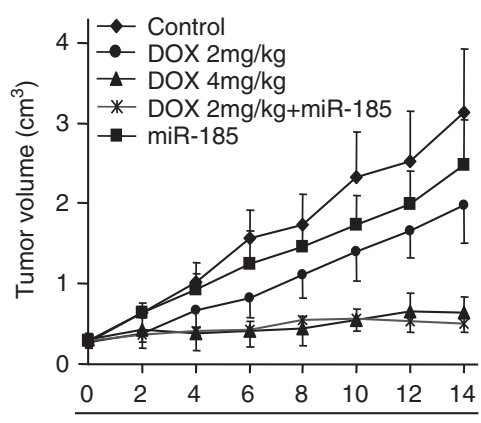

Days during therapeutic treatment

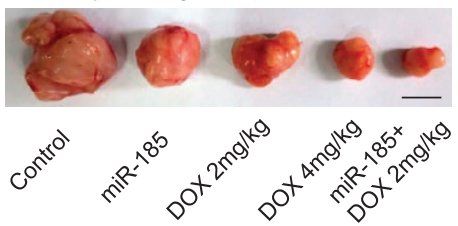

d

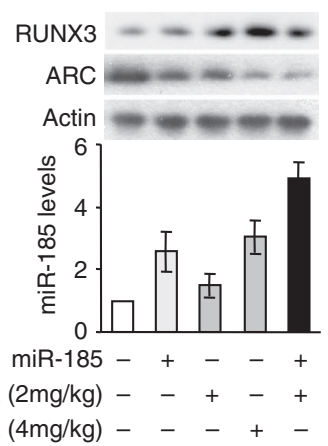

e

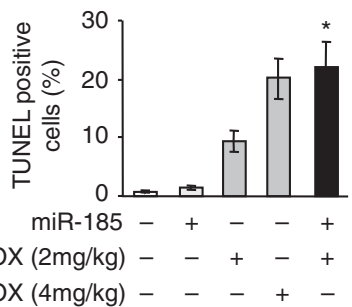

Figure 6 MiR-185 enhances the chemotherapeutic effect in vivo. (a) A total of $1 \times 10^{7}$ SGC-7901 cells stably overexpressing miR-185 or negative control were injected subcutaneously into BALB/c nude mice. Line graph shows tumor volumes measured every 3 days from day 7 . Photographs show morphology of tumors at day $34, n=5$ each group. (b) Immunoblot of ARC protein (top) and miR-185 expression levels (bottom) in generated xenograft tumors shown in a, ${ }^{*} P<0.05$ compared with control. (c) A total of $1 \times 10^{7}$ SGC-7901 cells were injected subcutaneously into BALB/c nude mice. When tumors reached $250-300 \mathrm{~mm}^{3}$, therapeutic treatment as indicated was given every other day. Tumor volumes were monitored during 2-week therapy ( $n=6$ each group) (top). At the end of this experiment, tumors were dissected and photographed. Representative images of tumors were shown (bottom), scale bar $=1 \mathrm{~cm}$. (d) Western blot analysis as indicated and miR-185 expression levels in the xenograft tumors described in $\mathbf{c}$. (e) Apoptosis detected in sections of xenograft tumors described in c, $n=6$ each group. ${ }^{*} P<0.05$ compared with doxorubicin (DOX) ( $2 \mathrm{mg} / \mathrm{kg}$ ) alone. Error bars represent S.D. TUNEL, terminal deoxynucleotidyl transferase dUTP nick-end labeling 
a
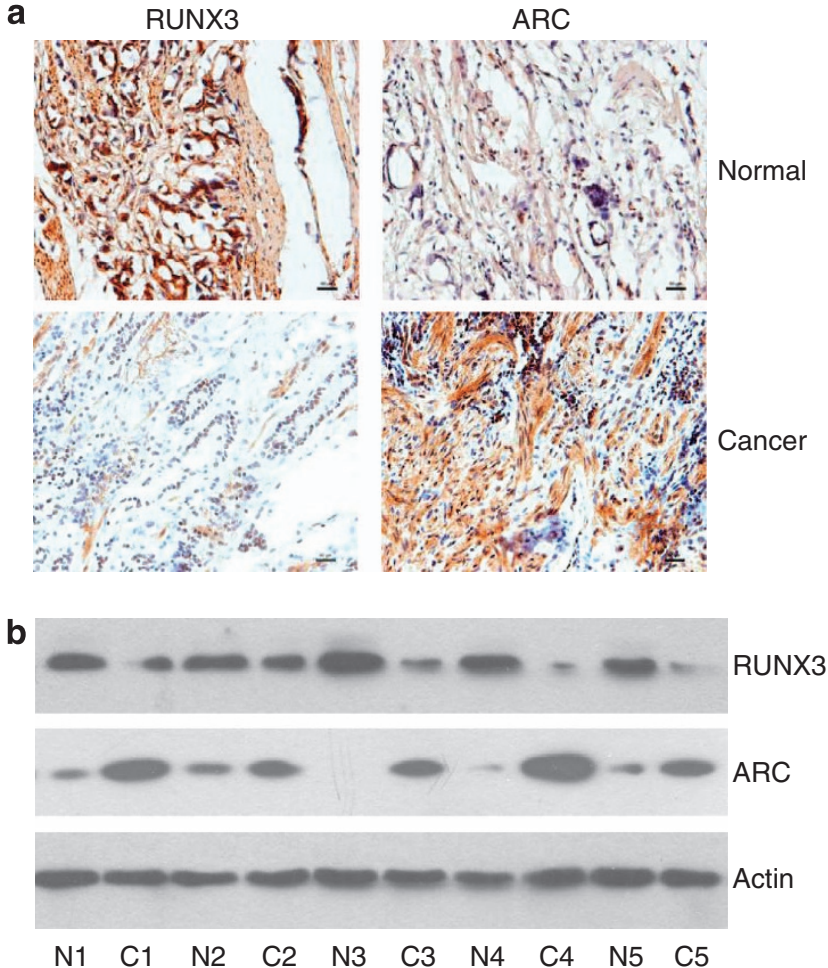

Figure 7 RUNX3 and ARC are dysregulated in human gastric carcinomas. (a) Immunohistochemistry staining of RUNX3 and ARC in paraffin sections of patient tissues, scale bar $=50 \mu \mathrm{m}$. (b) Immunoblot of RUNX3 and ARC proteins in five representative pairs of normal gastric tissues $(\mathrm{N})$ and matched cancer tissues (C). The patient numbers were 1-5 as in Supplementary Table 1

RUNX3 and ARC are dysregulated in human gastric carcinomas. To further explore the potential clinical relevance of miR-185-mediated signaling pathway in human gastric cancer, we determined the expression levels of RUNX3 and ARC in human gastric tissues (Supplementary Table 1). Immunohistochemical staining showed loss of RUNX3 expression and high expression of ARC in tumor tissues (Figure 7a). This observation was further confirmed by immunoblotting of RUNX3 and ARC protein in these clinical samples. Five representative results were shown here (Figure 7b). According to our data, the inverse association between ARC expression and RUNX3 protein levels was indicated. These findings are consistent with the results in gastric cancer cell lines, and suggest that RUNX3miR-185-ARC axis may be involved in the development of gastric cancer.

\section{Discussion}

Chemotherapy has an important role in the treatment of a variety of cancers, including gastric cancer. However, chemoresistance is a major challenge to effective treatment. It is quite urgent to explore potential mechanisms involved in chemotherapy resistance. Our current study identified miR-185 as a regulator in the sensitivity of gastric cancer cells to chemotherapy. We found that miR- 185 was expressed at a lower level in several gastric cancer cell lines and human gastric cancer tissues. Enforced expression of miR-185 led to enhanced sensitivity of gastric cancer cells to low-dose chemotherapeutic agents. Furthermore, we discovered an miR-185-controlled apoptotic pathway involving ARC, which was regulated by RUNX3. Our conclusion was also supported by the results in nude mice model and clinical gastric cancer specimens.

Aberrant miR-185 expression has been identified in various cancers in previous studies. It has been shown that miR-185 was abundant in hepatocellular carcinoma. ${ }^{29}$ However, there were opposite findings that decrease of miR-185 levels occurred in ovarian cancer, ${ }^{30,31}$ glioma $^{32}$ and prostate cancer. ${ }^{33}$ The function of miR-185 in cancer cells may be cell type-dependent. ${ }^{34}$ In gastric cancer, lower expression levels of miR-185 were observed, ${ }^{23,35}$ which was consistent with our findings. However, the exact function of miR-185 in gastric cancer is little known. Our results displayed miR-185 tumorsuppressing activity in gastric cancer xenograft model. Based on our experiments, induction of miR-185 could be a possible therapy strategy for gastric cancer.

MiR-185 suppressed the proliferation potential and tumor growth in colorectal cancer, ${ }^{28}$ in non-small-cell lung cancer ${ }^{24}$ and in ovarian cancer. ${ }^{31}$ It inhibited invasion and migration in human prostate cancer xenograft model. ${ }^{33}$ Moreover, miR185 could sensitize high Six1-expressing cancer cells to tumor necrosis factor-related apoptosis-inducing ligand (TRAIL)induced apoptosis. ${ }^{31}$ MiR-152 and miR-185 cocontributed to chemosensitivity through epigenetic regulation in ovarian cancer. ${ }^{34}$ These investigations implied that miR-185 was possibly related to apoptosis. However, the mechanism of miR-185 involvement in apoptosis signaling pathway is unclear. Our results showed that miR-185 increased anticancer drug-induced apoptosis by directly targeting ARC. MiR-185 has been shown to target other proteins in different types of cells. For instance, miR-185 regulated HIF- $2 \alpha$ in human umbilical vein endothelial cells. ${ }^{36}$ Six $1,{ }^{31}$ androgen receptor, ${ }^{33}$ Cdc42 and $\mathrm{RhoA}^{28}$ were proven to be downregulated by miR-185 in cancer cells. Interestingly, miR-185 could disrupt DNA methylation by targeting DNA methyltransferase 1 in ovarian cancer ${ }^{34}$ and gastric cancer. ${ }^{35}$ In this context, we clearly demonstrated that ARC is a direct target of miR-185. To the best of our knowledge, this is the first report on the regulation of ARC expression mediated by an miRNA. ARC was highly expressed in various cancer cells. The function of miR-185 in these ARC-abundant cancer cells needs to be further explored.

Because of the limited number of human gastric cancer tissues we collected in this study, we did not analyze the relevance of miR-185 expression levels to clinicopathologic features and prognosis of these patients. The clinical significance of miR-185 during gastric cancer progression needs to be investigated in the further studies. Besides, miR-185 polymorphic nucleotide variations were discovered in breast cancer, ${ }^{37}$ chronic lymphocytic leukemia and colorectal cancer. ${ }^{38}$ It would be interesting to identify whether miR-185 SNPs exists and functions in gastric cancer.

Our present data revealed that RUNX3 enhanced miR-185 expression at the transcriptional level in gastric cancer. It has been reported that RUNX3 increased Bim expression ${ }^{26}$ and decreased vascular endothelial growth factor levels ${ }^{39}$ owing 
to its transcriptional activity. Previous researches have reported that methylation modification was possibly related to RUNX3 expression level. ${ }^{22}$ MDM2-mediated ubiquitination participated in the regulation of RUNX3 as well. ${ }^{40}$ In this study, we observed upregulation of RUNX3 in human stomach cancer cells exposed to chemotherapeutic drugs. The mechanism by which RUNX3 was upregulated in response to anticancer agents remains to be determined.

The therapeutic effects of conventional anticancer agents were limited owing to chemoresistance and their toxicity. MiRNAs have been demonstrated to participate in cancer progression and resistance to chemotherapy, thereby acting as a potential candidate for therapeutic intervention. Anti-miR-21 in combination with S-TRAIL resulted in significantly increased apoptosis in murine glioma models. ${ }^{41}$ Administration of anti-miR-221 or anti-miR-181b along with tamoxifen led to apparent reduction in breast tumor size. ${ }^{42}$ Combination treatment of miR-200c and paclitaxel markedly decreased ovarian tumor burden. ${ }^{43}$ Here, we report that restoration of miR-185 alone can inhibit gastric cancer tumor growth. Moreover, combination therapy using enforced miR-185 expression and lower dose chemotherapeutic drugs had an effective therapeutic activity against large established tumors, with decreased host toxicity. Our results are first to implicate that miR-185 can be considered as a target for gastric cancer treatment. It would be quite possible to develop miR-185based combinatorial strategy for effective cancer therapy.

Taken together, we report here that miR-185 increases the chemosensitivity of gastric cancer cells in vitro and in vivo. It exerts tumor-suppressing function through negatively regulating ARC. Besides, miR-185 upregulation in response to cisplatin or doxorubicin treatment in gastric cancer cells is dependent on RUNX3 transcriptional activity. Our results suggest that miR-185 might be a novel therapeutic target for gastric cancer.

\section{Materials and Methods \\ Reagents and cell culture. Cisplatin, doxorubicin and G418 were purchased from Sigma (St. Louis, MO, USA). Anti-ARC antibody and anti-RUNX3 antibody were obtained from Abcam (Cambridge, UK). Anticleaved caspase-3 antibody was obtained from Cell Signaling Technology (Danvers, MA, USA). Human gastric cancer cell line SGC-7901 was obtained as we described previously. ${ }^{14}$ Human gastric epithelium cell, GES-1, and human gastric cancer cells, NCI-N87, MGC-803, BGC-823, AGS, were obtained from Beijing Institute for Cancer Research (Beijing, China). The cells were cultured in Dulbecco's modified Eagle's medium (GIBCO, Grand Island, NY, USA) supplemented with $10 \%$ fetal bovine serum, $100 \mathrm{U} / \mathrm{ml}$ penicillin and $100 \mu \mathrm{g} / \mathrm{ml}$ streptomycin in a humidified atmosphere containing $5 \% \mathrm{CO}_{2}$ at $37^{\circ} \mathrm{C}$.}

Clinical gastric cancer samples. Gastric cancer tissues and matched noncancerous gastric tissues from 25 patients (19 males and 6 females; mean age, 59.76 years, range, 44-81 years) with gastric cancer were collected from Beijing Military General Hospital (Beijing, China). These patients were randomly selected from the patient pool of the hospital's Gastrointestinal clinic; none of the patients received chemotherapy or radiotherapy. Human tissues were collected during gastroscopy and immediately frozen in liquid nitrogen. The study was approved by the ethics committee of the Beijing Military General Hospital. Informed consent was obtained from all study subjects.

Cell viability assay and TUNEL analysis. Cell death was determined by trypan blue exclusion. The trypan blue-positive and -negative cells were counted on a hemocytometer. TUNEL assay was performed using a kit from Roche Applied Science (Hamburg, Germany). The procedures were followed as per the instructions in the kit. The samples were imaged using a laser scanning confocal microscope (Zeiss LSM 510 META, Carl Zeiss, Jena, Germany).

Adenovirus. Adenovirus ARC and adenovirus $\beta$-galactosidase were as we described previously. ${ }^{14}$ Constructions of adenoviral miR-185 and RUNX3 were described in Supplementary Information. All adenoviruses were amplified in HEK293 cells. Adenoviral infection of cancer cells was performed as we described previously. ${ }^{14}$

Cell transfection with miRNA duplexes or miRNA inhibitors. The hsa-miR-185 duplexes were synthesized by GenePharma Co. Ltd (Shanghai, China). MiR-185 mimic sequence was, 5'-UGGAGAGAAAGGCAGUUCCUGA-3'. Mimic control sequence was, $5^{\prime}$-UUGUACUACACAAAAGUACUG-3'. Chemically modified antisense oligonucleotides (antagomirs) were used to inhibit endogenous miR-185 expression. The antagomir sequence was, $5^{\prime}$-UCAGGAACUGCCUUUC UCUCCA-3'. All the bases were 2'-O-methyl-modified (GenePharma Co. Ltd). The antagomir control sequence was, 5'-CAGUACUUUUGUGUAGUACAA-3' (GenePharma Co. Ltd). Cells were transfected with miRNA duplexes $(50 \mathrm{nM})$ or antagomirs (50 nM) using Lipofectamine 2000 (Invitrogen, Grand Island, NY, USA) according to the manufacturer's instructions.

Reporter constructions and luciferase assay. The fragment of ARC $3^{\prime}$-UTR containing two miR-185 binding sites (sites 1 and 2) was amplified by PCR. The forward primer was $5^{\prime}$-ATGCTGCTGGAGCTGAATCGGAT-3', and the reverse primer was $5^{\prime}$-ATAGAGCGCCTTTTGTGTAAGTC- ${ }^{\prime}$. To generate reporter vector containing miR-185 binding sites, the PCR product was cloned downstream of the stop codon of the luciferase gene of pGL3 vector (Promega, Madison, WI, USA). To generate ARC $3^{\prime}$-UTR-Mut1 and ARC $3^{\prime}$-UTR-Mut2, the mutations (the wild-type ARC $3^{\prime}$-UTR site1: TCTCTCCA, ARC $3^{\prime}$-UTR-Mut1: TCAGGACA; the wild-type ARC $3^{\prime}$-UTR site 2: TCTCTCCA, ARC $3^{\prime}$-UTR-Mut2: TCAGGACA) were produced using the QuikChange II XL Site-Directed Mutagenesis Kit (Stratagene, La Jolla, CA, USA). The two binding sites of miR-185 were mutated simultaneously to generate ARC $3^{\prime}$-UTR-Mut1 +2 .

For luciferase assay performed in HEK-293 cells, cells in 24-well plates were co-transfected with $200 \mathrm{ng}$ per well luciferase reporter constructs, $400 \mathrm{ng}$ per well miR-185 mimic or mimic control using Lipofectamine 2000 (Invitrogen). SV-Renilla luciferase plasmids of $5 \mathrm{ng}$ per well served as the internal control. Cells were harvested at $24 \mathrm{~h}$ after transfection and the luciferase activity was detected using the Dual Luciferase Reporter Assay Kit (Promega) according to the manufacturer's instructions. A measure of $30 \mu \mathrm{l}$ of protein samples was analyzed in a luminometer. Firefly luciferase activities were normalized to Renilla luciferase activity. The similar strategy was used to perform luciferase analysis in SGC-7901 cells.

The miR-185 promoter region (1297 bp) was amplified from human genomic DNA to generate wild-type promoter. The fragment containing three RUNX3 putative binding sites was amplified using the forward primer, $5^{\prime}$-GGGCAGAGCA GAGCTACAAATG- ${ }^{\prime}$, and the reverse primer, $5^{\prime}$-AACCCAGCTCTAGCCAG CAGGT-3'. The PCR product was cloned into the reporter vector pGL4.17 (Promega). The introduction of mutations in three potential RUNX3 binding sites (wild-type: TGTGGT $\rightarrow$ mutated: TACACT) was carried out using the QuikChange II XL Site-Directed Mutagenesis Kit (Stratagene). Luciferase assay was performed as described. $^{44}$

Immunoblotting. Immunoblotting was performed as we described. ${ }^{14}$ Briefly, cells were lysed for $1 \mathrm{~h}$ at $4{ }^{\circ} \mathrm{C}$ in a lysis buffer $(20 \mathrm{mmol} / \mathrm{l}$ Tris $(\mathrm{pH} 7.5)$, $2 \mathrm{mmo} / / \mathrm{EDTA}, 3 \mathrm{mmol} / / \mathrm{EGTA}, 2 \mathrm{mmol} / / \mathrm{DTT}, 250 \mathrm{mmol} / \mathrm{l}$ sucrose, $0.1 \mathrm{mmol} / \mathrm{l}$ phenylmethylsulfonyl fluoride, $1 \%$ Triton X-100) containing a protease inhibitor cocktail. Protein samples were subjected to $12 \%$ SDS-PAGE and transferred to nitrocellulose membranes. Blots were probed using corresponding primary antibodies. Then, the horseradish peroxidase-conjugated secondary antibodies were used. Antigen-antibody complexes were tested by enhanced chemiluminescence.

qRT-PCR. Stem-loop qRT-PCR was carried out as described on an Applied Biosystems ABI Prism 7000 sequence detection system (Applied Biosystems, Carlsbad, CA, USA). ${ }^{44}$ Total RNA was extracted using Trizol reagent. After DNase I (Takara, Japan) treatment, RNA was reverse transcribed with Reverse Transcriptase Kit (Toyobo, Osaka, Japan). Mature miR-185 levels were measured using SYBR Green Real-Time PCR Master Mix (Toyobo) according to the manufacturer's instructions. The sequences of miR-185 primers were: forward, 
5'-CATGGAGAGAAAGGCAGT-3'; reverse, $\quad 5^{\prime}$-GTGCAGGGTCCGAGGT-3' The levels of miR-185 analyzed by qRT-PCR were normalized to that of U6. The sequences of U6 primers were: forward, $5^{\prime}$-CTCGCTTCGGCAGCACA-3'; reverse, $5^{\prime}$-AACGCTTCACGAATTTGCGT- $3^{\prime}$. Quantitative detection of ARC and RUNX3 was performed using the same strategy. The primers used for ARC were: forward, 5'-CGAGTCCGAAGATTCCTGA-3'; reverse, 5'-GACCCTCCGGAGTT TATTCA-3'. The primers used for RUNX3 were: forward, $5^{\prime}$-GACAGCCC CAACTTCCTCT-3'; reverse, 5'-CACAGTCACCACCGTACCAT-3'. The mRNAs levels were normalized to that of glyceraldehyde-3-phosphate dehydrogenase (GAPDH). The sequences of GAPDH primers were: forward, $5^{\prime}$-GTCGGAGTC

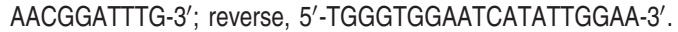

ChIP Analysis. ChIP was carried out as we described previously. ${ }^{45}$ Briefly, cells were washed two times with phosphate-buffered saline (PBS), and then were incubated for $10 \mathrm{~min}$ with $4 \%$ formaldehyde at room temperature. After washing two times with PBS, cells were lysed in a lysis buffer for $1 \mathrm{~h}$ at $4{ }^{\circ} \mathrm{C}$. The cell lysates were sonicated into chromatin fragments $(500-800 \mathrm{bp}$ length). The samples were precleared with Protein A-agarose (Roche Applied Science) at $4^{\circ} \mathrm{C}$. The anti-RUNX3 antibody or anti-actin antibody was added and rocked overnight. Immunoprecipitates were captured with $10 \%$ (V/v) Protein A-agarose. To analyze RUNX3 binding to the promoter of miR-185, PCRs were carried out using primers that encompass RUNX3 BS1 or BS2 or BS3 of the miR-185 promoter. The primers were: BS1 (forward, 5'-GGACTCTTGAGATGAGATCAC-3'; reverse, 5'-CTCTCCATTCTGTCAACTGG-3'); BS2 (forward, $5^{\prime}$-TCCTGCAGATGTTCA GATGC-3'; reverse, 5'-CAACTCTCAGCATGTCCTTG-3'); BS3 (forward, 5'-C CAGATCAAGATATGGTGACC-3'; reverse, 5'-ACCTGTGACCTTGCCTTTG-3').

Subcutaneous tumor xenograft model. For the experiments in Figure $6 \mathrm{a}$, approximately $1 \times 10^{7}$ SGC-7901 cells, established stable SGC7901-miR-185 or SGC-7901-negative control cells, were injected subcutaneously into the right flanks of female BALB/c nude mice (4-5 weeks old, 5 mice per group).The tumor size was measured with a caliper every 3 days. The tumor volume was calculated using the formula volume $=$ length $\times$ width $^{2} / 2$. The mice were killed at day 34 , and the tumors were separated for further analysis. For the combination therapy experiments in Figure 6c, $1 \times 10^{7}$ SGC-7901 cells were injected subcutaneously into the right flanks of female BALB/C nude mice (4-5 weeks old). When tumors reached an average volume of $250-300 \mathrm{~mm}^{3}$, the mice were randomly divided into five groups, six mice in each group. According to the experimental design, intraperitoneal delivery of doxorubicin and/or intratumoral injection of miR-185 adenovirus was administered every other day. Two different doses of doxorubicin (2 and $4 \mathrm{mg} / \mathrm{kg}$ ) were used. During doxorubicin and/or miR-185 adenovirus treatment in established tumors, the tumor size and body weight of the mice were monitored every other day. At the end of the experiment, the mice were killed and the tumors were separated for further analysis. Animal experiments were reviewed and approved by the Animal Care Committee, Institute of Zoology, Chinese Academy of Sciences.

Statistical analysis. All statistical analyses were performed using the SPSS 13.0 statistical software package. The results are expressed as means \pm S.D. of at least three independent experiments. The differences among experimental groups were evaluated by one-way analysis of variance. Paired data were determined by two-tailed Student's $t$-test. $P<0.05$ was considered statistically significant.

Additional Materials and Methods are available in Supplementary Information.

\section{Conflict of Interest}

The authors declare no conflict of interest.

Acknowledgements. This work was supported by National Natural Science Foundation of China (81170109), and a grant from ACS (120861).

1. Milne $A N$, Carneiro $F, O$ 'morain $C$, Offerhaus $G$. Nature meets nurture: molecular genetics of gastric cancer. Hum Genet 2009; 126: 615-628.

2. Jordan $\mathrm{P}$, Carmo-Fonseca M. Molecular mechanisms involved in cisplatin cytotoxicity. Cell Mol Life Sci 2000; 57: 1229-1235.

3. Longley D, Johnston P. Molecular mechanisms of drug resistance. J Pathol 2005; 205 275-292.
4. Zhao Y, Samal E, Srivastava D. Serum response factor regulates a muscle-specific microRNA that targets Hand2 during cardiogenesis. Nature 2005; 436: 214-220.

5. Calin GA, Croce CM. MicroRNA signatures in human cancers. Nat Rev Cancer 2006; 6: 857-866

6. Bonci D, Coppola V, Musumeci M, Addario A, Giuffrida R, Memeo L et al. The miR-15amiR-16-1 cluster controls prostate cancer by targeting multiple oncogenic activities. Nat Med 2008; 14: 1271-1277.

7. Ma L, Teruya-Feldstein J, Weinberg RA. Tumour invasion and metastasis initiated by microRNA-10b in breast cancer. Nature 2007; 449: 682-688.

8. Garofalo M, Romano G, Di Leva G, Nuovo G, Jeon Y-J, Ngankeu A et al. EGFR and MET receptor tyrosine kinase-altered microRNA expression induces tumorigenesis and gefitinib resistance in lung cancers. Nat Med 2011; 18: 74-82.

9. Hatley ME, Patrick DM, Garcia MR, Richardson JA, Bassel-Duby R, Van Rooij E et al. Modulation of K-Ras-dependent lung tumorigenesis by microRNA-21. Cancer Cell 2010; 18: 282-293.

10. Koseki T, Inohara N, Chen S, Núñez G. ARC, an inhibitor of apoptosis expressed in skeletal muscle and heart that interacts selectively with caspases. Proc Natl Acad Sci 1998; 95: 5156-5160.

11. Ludwig-Galezowska AH, Flanagan L, Rehm M. Apoptosis repressor with caspase recruitment domain, a multifunctional modulator of cell death. J Cell Mol Med 2011; 15: 1044-1053.

12. Mercier I, Vuolo M, Jasmin J-F, Medina CM, Williams M, Mariadason JM et al. ARC (apoptosis repressor with caspase recruitment domain) is a novel marker of human colon cancer. Cell Cycle 2008; 7: 1640-1647.

13. Mercier I, Vuolo M, Madan R, Xue X, Levalley A, Ashton A et al. ARC, an apoptosis suppressor limited to terminally differentiated cells, is induced in human breast cancer and confers chemo-and radiation-resistance. Cell Death Differ 2005; 12: 682-686.

14. Wang J-X, Li Q, Li P-F. Apoptosis repressor with caspase recruitment domain contributes to chemotherapy resistance by abolishing mitochondrial fission mediated by dynaminrelated protein-1. Cancer Res 2009; 69: 492-500.

15. Li P-F, Li J, Müller E-C, Otto A, Dietz R, von Harsdorf R. Phosphorylation by protein kinase CK2: a signaling switch for the caspase-inhibiting protein ARC. Mol Cell 2002; 10 : 247-258.

16. Tan W-Q, Wang J-X, Lin Z-Q, Li Y-R, Lin Y, Li P-F. Novel cardiac apoptotic pathway: the dephosphorylation of apoptosis repressor with caspase recruitment domain by calcineurin. Circulation 2008; 118: 2268-2276.

17. Li Y-Z, Lu D-Y, Tan W-Q, Wang J-X, Li P-F. P53 initiates apoptosis by transcriptionally targeting the antiapoptotic protein ARC. Mol Cell Biol 2008; 28: 564-574.

18. Lu D, Liu J, Jiao J, Long B, Li Q, Tan W et al. Transcription factor Foxo3a prevents apoptosis by regulating calcium through the apoptosis repressor with caspase recruitment domain. J Biol Chem 2013; 288: 8491-8504.

19. Foo RS-Y, Chan LK, Kitsis RN, Bennett MR. Ubiquitination and degradation of the antiapoptotic protein ARC by MDM2. J Biol Chem 2007; 282: 5529-5535.

20. Carter BZ, Qiu YH, Zhang N, Coombes KR, Mak DH, Thomas DA et al. Expression of ARC (apoptosis repressor with caspase recruitment domain), an antiapoptotic protein, is strongly prognostic in AML. Blood 2011; 117: 780-787.

21. Braun T, Woollard A. RUNX factors in development: Lessons from invertebrate model systems. Blood Cells Mol Dis 2009; 43: 43-48.

22. Li Q-L, Ito K, Sakakura C, Fukamachi H, Inoue $\mathrm{K}-\mathrm{i}$, Chi X-Z et al. Causal relationship between the loss of RUNX3 expression and gastric cancer. Cell 2002; 109: 113.

23. Volinia S, Calin GA, Liu C-G, Ambs S, Cimmino A, Petrocca F et al. A microRNA expression signature of human solid tumors defines cancer gene targets. Proc Natl Acad Sci USA 2006; 103: 2257-2261.

24. Takahashi Y, Forrest AR, Maeno E, Hashimoto T, Daub CO, Yasuda J. MiR-107 and MiR185 can induce cell cycle arrest in human non small cell lung cancer cell lines. PLoS One 2009; 4: e6677.

25. Medina-Ramirez CM, Goswami S, Smirnova T, Bamira D, Benson B, Ferrick N et al. Apoptosis inhibitor ARC promotes breast tumorigenesis, metastasis, and chemoresistance. Cancer Res 2011; 71: 7705-7715.

26. Yano T, Ito K, Fukamachi H, Chi X-Z, Wee H-J, Inoue K-i et al. The RUNX3 tumor suppressor upregulates bim in gastric epithelial cells undergoing transforming growth factor $\beta$-induced apoptosis. Mol Cell Biol 2006; 26: 4474-4488.

27. Yamada C, Ozaki T, Ando K, Suenaga $Y$, Inoue K-i, Ito $Y$ et al. RUNX3 modulates DNA damage-mediated phosphorylation of tumor suppressor p53 at Ser-15 and acts as a coactivator for p53. J Biol Chem 2010; 285: 16693-16703.

28. Liu M, Lang N, Chen X, Tang Q, Liu S, Huang J et al. miR-185 targets RhoA and Cdc42 expression and inhibits the proliferation potential of human colorectal cells. Cancer Lett 2011; 301: 151-160.

29. Budhu A, Jia HL, Forgues M, Liu CG, Goldstein D, Lam A et al. Identification of metastasisrelated microRNAs in hepatocellular carcinoma. Hepatology 2008; 47: 897-907.

30. Wyman SK, Parkin RK, Mitchell PS, Fritz BR, O'Briant K, Godwin AK et al. Repertoire of microRNAs in epithelial ovarian cancer as determined by next generation sequencing of small RNA cDNA libraries. PLoS One 2009; 4: e5311.

31. Imam J, Buddavarapu K, Lee-Chang J, Ganapathy S, Camosy C, Chen Y et al. MicroRNA185 suppresses tumor growth and progression by targeting the Six1 oncogene in human cancers. Oncogene 2010; 29: 4971-4979. 
32. Tang H, Wang Z, Liu X, Liu Q, Xu G, Li G et al. LRRC4 inhibits glioma cell growth and invasion through a miR-185-dependent pathway. Curr Cancer Drug Targets 2012; 12: 1032-1042.

33. Qu F, Cui X, Hong Y, Wang J, Li Y, Chen $L$ et al. MicroRNA-185 suppresses proliferation, invasion, migration, and tumorigenicity of human prostate cancer cells through targeting androgen receptor. Mol Cell Biochem 2013; 377: 1-10.

34. Xiang $\mathrm{Y}, \mathrm{Ma} \mathrm{N}$, Wang D, Zhang $\mathrm{Y}$, Zhou J, Wu G et al. MiR-152 and miR-185 co-contribute to ovarian cancer cells cisplatin sensitivity by targeting DNMT1 directly: a novel epigenetic therapy independent of decitabine. Oncogene 2013; 33: 378-386.

35. Yoon JH, Choi YJ, Choi WS, Ashktorab H, Smoot D, Nam SW et al. GKN1-miR-185DNMT1 axis suppresses gastric carcinogenesis through regulation of epigenetic alteration and cell cycle. Clin Cancer Res 2013; 19: 4599-4610.

36. Ho JD, Metcalf JL, Yan MS, Turgeon PJ, Wang JJ, Chalsev M et al. Functional importance of Dicer protein in the adaptive cellular response to hypoxia. J Biol Chem 2012; 287: 29003-29020.

37. Farazi TA, Horlings HM, Jelle J, Mihailovic A, Halfwerk H, Morozov P et al. MicroRNA sequence and expression analysis in breast tumors by deep sequencing. Cancer Res 2011; 71: 4443-4453.

38. Wojcik SE, Rossi S, Shimizu M, Nicoloso MS, Cimmino A, Alder H et al. Non-codingRNA sequence variations in human chronic lymphocytic leukemia and colorectal cancer. Carcinogenesis 2010; 31: 208-215.

39. Peng Z, Wei D, Wang L, Tang H, Zhang J, Le X et al. RUNX3 inhibits the expression of vascular endothelial growth factor and reduces the angiogenesis, growth, and metastasis of human gastric cancer. Clin Cancer Res 2006; 12: 6386-6394.

40. Chi X-Z, Kim J, Lee Y-H, Lee J-W, Lee K-S, Wee H et al. Runt-related transcription factor RUNX3 is a target of MDM2-mediated ubiquitination. Cancer Res 2009; 69: 8111-8119.
41. Corsten MF, Miranda R, Kasmieh R, Krichevsky AM, Weissleder R, Shah K. MicroRNA-21 knockdown disrupts glioma growth in vivo and displays synergistic cytotoxicity with neural precursor cell-delivered S-TRAIL in human gliomas. Cancer Res 2007; 67: 8994-9000.

42. Lu Y, Roy S, Nuovo G, Ramaswamy B, Miller T, Shapiro C et al. Anti-microRNA-222 (anti-miR-222) and-181B suppress growth of tamoxifen-resistant xenografts in mouse by targeting TIMP3 protein and modulating mitogenic signal. J Biol Chem 2011; 286: $42292-42302$.

43. Cittelly DM, Dimitrova I, Howe EN, Cochrane DR, Jean A, Spoelstra NS et al. Restoration of miR-200c to ovarian cancer reduces tumor burden and increases sensitivity to paclitaxel. Mol Cancer Therap 2012; 11: 2556-2565.

44. Lin Z, Murtaza I, Wang K, Jiao J, Gao J, Li P-F. miR-23a functions downstream of NFATc3 to regulate cardiac hypertrophy. Proc Natl Acad Sci USA 2009; 106: 12103-12108.

45. Wang J-X, Jiao J-Q, Li Q, Long B, Wang K, Liu J-P et al. miR-499 regulates mitochondrial dynamics by targeting calcineurin and dynamin-related protein-1. Nat Med 2010; 17: 71-78.

(i) (-) $\odot$ Cell Death and Disease is an open-access journal published by Nature Publishing Group. This work is licensed under a Creative Commons Attribution-NonCommercialNoDerivs 3.0 Unported License. The images or other third party material in this article are included in the article's Creative Commons license, unless indicated otherwise in the credit line; if the material is not included under the Creative Commons license, users will need to obtain permission from the license holder to reproduce the material. To view a copy of this license, visit http://creativecommons.org/licenses/ by-nc-nd/3.0/

Supplementary Information accompanies this paper on Cell Death and Disease website (http://www.nature.com/cddis) 